\title{
Longitudinal and transverse spin relaxation times of magnetic single adatoms: An $a b$ initio analysis
}

\author{
Julen Ibañez-Azpiroz, Manuel dos Santos Dias, Stefan Blügel, and Samir Lounis \\ Peter Grünberg Institute and Institute for Advanced Simulation, Forschungszentrum Jülich \& JARA, D-52425 Jülich, Germany \\ (Received 1 August 2017; revised manuscript received 20 September 2017; published 6 October 2017)
}

\begin{abstract}
We present a systematic ab initio investigation of the longitudinal and transverse spin relaxation times of magnetic single adatoms deposited on metallic substrates. Our analysis based on time-dependent density functional theory shows that the longitudinal time $T_{\|}$is of order femtosecond while the transverse time $T_{\perp}$ is of order picosecond, i.e. $T_{\perp} \gg T_{\|}$. This comes as a consequence of the different energy scales of the corresponding processes: $T_{\|}$involves spin-density excitations of order $\mathrm{eV}$, while $T_{\perp}$ is governed by atomic spin excitations of order meV. Comparison to available inelastic scanning tunneling spectroscopy $d I / d V$ experimental curves shows that the order of magnitude of $T_{\perp}$ agrees well with our results. Regarding $T_{\|}$, the time scale calculated here is several orders of magnitude faster than what has been measured up to now; we therefore propose that an ultrafast laser pulse measuring technique is required in order to access the ultrafast spin dynamics described in this paper.
\end{abstract}

DOI: 10.1103/PhysRevB.96.144410

\section{INTRODUCTION}

Single adatoms deposited on substrates offer an exceptional scenario for studying magnetism at the atomic scale, given that these tiny objects can develop a large magnetic moment of several Bohr magnetons [1-3] as well as a large magnetic anisotropy energy barrier of few meV [4-12], both extremely desirable properties for potential applications in spintronic devices. Interestingly, the possibility of tuning and engineering these and other properties by the suitable combination of adatom and substrate material (possibly including coating layers) provides plenty of room for research in this area.

In order to achieve the ultimate goal of a technologically applicable magnetic single adatom, however, not only the static properties need to be adequate but also the dynamical ones, and in particular the ones related to the spin, i.e., the spin dynamics. For example, fast spin dynamics can be useful when the goal is to transfer magnetic information from or to the adatom, while slow spin dynamics are desirable if the aim is to store magnetic information. In comparison to the static case, the study of spin dynamics of single adatoms is much more recent and has only hatched out after the advent of spin-polarized scanning tunneling microscopy (STM) and inelastic electron tunneling spectroscopy (IETS). These experimental techniques, occasionally used in combination with $\mathrm{x}$-ray magnetic circular dichroism (XMCD) [13] and electron paramagnetic resonance (EPR) [14], allow us to monitor the dynamical regime by, e.g., measuring atomic spin excitations [7,15-17] and quasiparticle interferences [18], accessing spin relaxation times [14,19] and even resolving highly dynamical processes like the reading and writing of magnetic information into a single adatom [20].

From the theoretical point of view, spin dynamics of single adatoms have also attracted a great deal of attention in the past few years. In this context, time-dependent density functional theory [21] (TDDFT) has proven to be a powerful tool for characterizing the spin-excitation spectrum and, more generally, giving insight into the connection between what is measured experimentally and the underlying electronic structure (see, e.g., Refs. [22-27]). Alongside, model Hamiltonians have also been used to analyze, among other aspects, the role of symmetry on the switching rate of the magnetic moment $[28,29]$, electron tunneling processes in IETS experiments [30-34] and spin decoherence [12,35,36].
In this paper, we present an ab initio study based on density functional theory (DFT) and TDDFT of two relaxation processes of single adatoms, namely the longitudinal and transverse spin relaxations characterized by the relaxation times $T_{\|}$and $T_{\perp}$, respectively. Physically, $T_{\|}$characterizes the relaxation of the size of the adatom's spin magnetic moment while $T_{\perp}$ describes its damped precessional motion. Employing $a b$ initio-derived expressions, we systematically provide hard numbers for $T_{\|}$and $T_{\perp}$ for a series of $3 d$ and $4 d$ transition metal adatoms deposited on two metallic substrates, namely $\mathrm{Ag}(100)$ and $\mathrm{Cu}(111)$. Our analysis shows that, while $T_{\|}$is of the order of femtosecond, $T_{\perp}$ ranges from a few to thousands of picoseconds, i.e., $T_{\perp} \gg T_{\|}$. Noteworthily, these time scales are settled by the corresponding energy scales of the associated processes: continuous spin-conserving singleparticle excitations of energy $\mathrm{eV}$ in the case of $T_{\|}$, atomic spin-flip spin excitations of energy $\mathrm{meV}$ in the case of $T_{\perp}$. In comparison to available experimental measurements, the relaxation times $T_{\perp}$ extracted from IETS $d I / d V$ curves show overall the same order of magnitude as the ones calculated in our work and agree remarkably well in specific cases such as $\mathrm{Fe}$ on $\mathrm{Cu}(111)$ [17]. Regarding $T_{\|}$, the time resolution of the currently available measuring techniques ranges from a few nanoseconds to hundreds of picoseconds [19,37], hence not enough to monitor the femtosecond regime predicted here. However, considering the technological developments within this field [38-40], access to the fs time scale of magnetic adatoms could be realized in the near future, thus giving access to the spin dynamics described in this paper.

The paper is organized as follows. Section II summarizes the technical details of the formalism used throughout the paper. In Sec. III we present DFT calculations of ground state properties of several $3 d$ and $4 d$ transition metal adatoms deposited on $\mathrm{Ag}(100)$ and $\mathrm{Cu}(111)$. In Sec. IV we extend the analysis to the dynamical regime; in particular, we calculate longitudinal (Sec. IV B 1) and transverse (Sec. IV B) relaxation times within the TDDFT framework. Conclusions and a summary of the main results are provided in Sec. V. In Appendices A and $\mathrm{B}$ we derive the connection between TDDFT and phenomenological models for the longitudinal and transverse dynamics, respectively. Finally, Appendix C contains a short summary of the Bloch-Redfield formalism in 

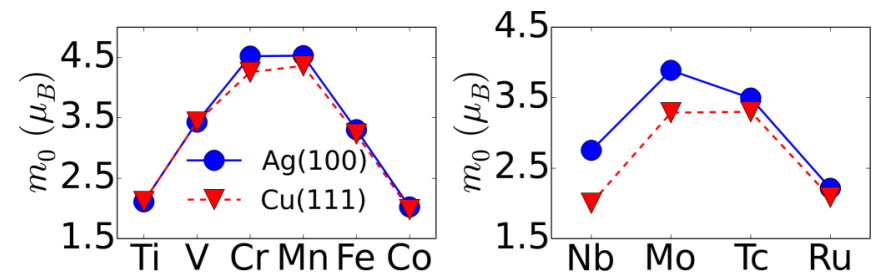

FIG. 1. Calculated ground state spin magnetic moments for several $3 d$ (left) and $4 d$ (right) transition metal adatoms deposited on $\mathrm{Ag}(100)$ and $\mathrm{Cu}(111)$ denoted by circles (blue) and triangles (red), respectively.

order to allow comparison of our TDDFT-based work to other theoretical analyses.

\section{COMPUTATIONAL DETAILS}

We have performed DFT calculations using the KorringaKohn-Rostoker Green function (KKRGF) approach, employing the atomic sphere approximation with full charge density [41] including spin-orbit coupling [24] (SOC). Exchange and correlation (XC) effects have been taken into account using the local spin-density approximation with the parametrization by Vosko, Wilk, and Nusair [42]. We have modeled the two surfaces $\mathrm{Ag}(100)$ and $\mathrm{Cu}(111)$ using a slab composed of 24 layers and augmented by two vacuum regions of $21.1 \AA$ thickness each, employing the lattice constants $a=5.46 \AA$ and $a=4.83 \AA$, respectively. The vertical distance from adatom to the surface layer has been calculated using the structural relaxation scheme implemented in the QUANTUM-ESPRESSO package [43], considering the convergence criterion whereby forces are $<10^{-4}$ Ry a.u. ${ }^{-1}$ and employing norm-conserving pseudopotentials, a $4 \times 4$ two-dimensional unit cell, $\Gamma$ point calculation, and a cutoff energy of $80 \mathrm{Ry}$. In all cases, the distance between adatom and substrate was reduced by approximately $15 \%$ with respect to the ideal value. Hence, for the sake of comparison, we adopted the same distance for all adatoms in the DFT and TDDFT calculations using the KKRGF method. Noteworthily, this method allows a real-space treatment of the adatoms through an embedding technique [41]. Following this scheme, we have employed converged real-space clusters of 43 and 55 sites for the $\operatorname{Ag}(100)$ and $\mathrm{Cu}(111)$ surfaces, respectively.

\section{GROUND STATE PROPERTIES}

In this section we analyze two ground state properties, namely the spin magnetic moment, denoted by $m_{0}$, and electronic density of states (DOS) of several $3 d$ and $4 d$ transition metal adatoms deposited on the metallic substrates $\operatorname{Ag}(100)$ and $\mathrm{Cu}(111)$. Let us begin with Fig. 1, where the calculated $m_{0}$ is depicted. This figure shows that all the considered adatoms develop large magnetic moments of more than $2 \mu_{B}$. Furthermore, $m_{0}$ acquires noninteger values, indicating the itinerant character of the adatom's $d$ electrons induced by the hybridization with the electrons of the metallic substrate. This feature is confirmed by the DOS, which is displayed in Fig. 2 for the specific case of $3 d$ adatoms deposited on $\operatorname{Ag}(100)$. This figure shows that the $d$-state peaks,

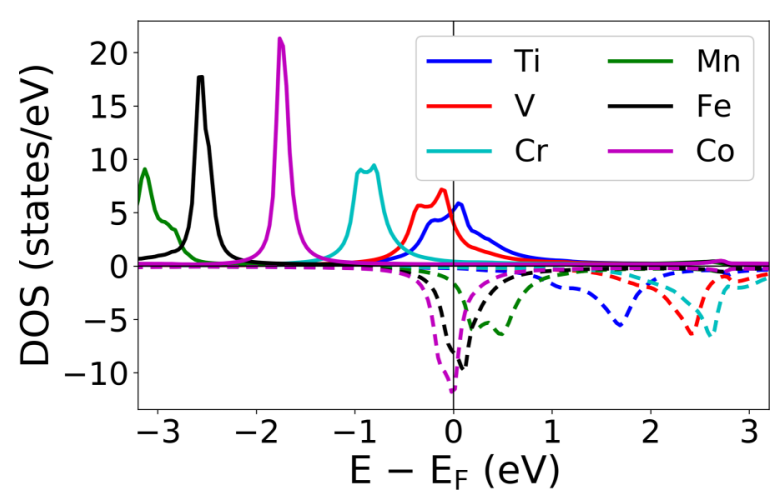

FIG. 2. Atom-projected total electronic DOS for $3 d$ adatoms deposited on $\operatorname{Ag}(100)$. The majority and minority spin channels are denoted by solid (positive) and dashed (negative) lines, respectively. The vertical line denotes the Fermi level.

so-called virtual bound states, are substantially broadened (between $\sim 0.1 \mathrm{eV}$ and $\sim 1 \mathrm{eV}$ depending on the adatom), which is a well-known consequence of hybridization with the substrate [2,17,24]. A further property indicated by Fig. 1 is that the first of the atomic Hund's rules is closely fulfilled, i.e., the half filled $d$-shell elements develop the largest magnetic moments, case of $\mathrm{Cr}$ and $\mathrm{Mn}$ for $3 d$, Mo and Tc for $4 d$. Finally, Fig. 1 shows that the choice of metallic substrate and surface orientation does not substantially affect the spin magnetic moment developed by the adatom, indicating that the symmetry of the substrate plays a minor role in this context. These ground state properties are consistent with the original works by Dederichs and co-workers [1-3], as well as with more recent studies [22-27].

We note that correlation effects beyond standard LDA, which can be non-negligible in some cases [44], can be incorporated into DFT by the use of a Hubbard $U$ term [45]. The main effect of this procedure would be to shift the $d$-state DOS peaks to higher or lower energies, thus modifying also the contribution at the Fermi level. In this work we do not adopt such a correction scheme since the present level of theory is qualitatively and often quantitatively correct as compared to experiments [22-27].

\section{SPIN SUSCEPTIBILITIES AND RELAXATION TIMES}

In this section we analyze dynamical properties of the magnetic adatoms studied in the previous section, paying special attention to relaxation times and their connection to the electronic structure. For this, let us consider the linear response of a ferromagnetic system to an externally applied time-dependent perturbation,

$$
\delta \mathbf{m}(\mathbf{r} ; t)=\int d \mathbf{r}^{\prime} \int d t^{\prime} \chi\left(\mathbf{r}, \mathbf{r}^{\prime} ; t-t^{\prime}\right) \delta \mathbf{V}\left(\mathbf{r}^{\prime} ; t^{\prime}\right) .
$$

Above, $\delta \mathbf{m}=\left(\delta m_{x}, \delta m_{y}, \delta m_{z}, \delta n\right) \quad$ and $\delta \mathbf{V}=\left(\delta B_{x}, \delta B_{y}\right.$, $\left.\delta B_{z}, \delta V\right)$, with $\delta m_{i}$ and $\delta B_{i}$, respectively, the components of the spin magnetic moment and external magnetic field, while $\delta n$ and $\delta V$ are the charge density and external scalar field, respectively. In frequency space and defining atomiclike quantities by integrating out the spatial dependence over 
atomic sites [23], the above expression takes the simplified form

$$
\delta \mathbf{m}(\omega)=\chi(\omega) \delta \mathbf{V}(\omega) .
$$

The quantity $\chi$ in the above equations is a $4 \times 4$ tensor that couples in general all components of the spin and charge responses with each other. If SOC is weak, however, the full response decouples into a longitudinal and transverse part [46]. This approximation is justified for the systems investigated here since the off-diagonal sectors of the susceptibility tensor are small in comparison to the diagonal ones. Then, assuming that the perturbation is purely of magnetic origin (i.e., $\delta V=0$ ), the change of the spin magnetic moment length is described by

$$
\delta m_{z}(\omega)=\chi_{\|}(\omega) \delta B_{z}(\omega) .
$$

Above, $\chi_{\|}(\omega)$ denotes the longitudinal spin susceptibility. This quantity is determined by excitations between electrons with same spin state, given that it involves the Pauli matrix $\sigma_{z}$ that is diagonal in spin basis [47]. On the other hand, the change of the transverse spin components can be compactly described using the circular combinations $m_{ \pm}=m_{x} \pm i m_{y}$ and $B_{ \pm}=B_{x} \pm i B_{y}$, yielding for the + component

$$
\delta m_{+}(\omega)=\chi_{ \pm}(\omega) \delta B_{+}(\omega) .
$$

Above, $\chi_{ \pm}(\omega)$ denotes the transverse spin susceptibility which, contrary to $\chi_{\|}(\omega)$, is determined by transitions that flip the spin state of the electrons due to the transverse Pauli spin matrices involved, which are off-diagonal in spin space [22-24]. In the following, the analysis is divided in two subsections: Sec. IV B 1 deals with the longitudinal response while Sec. IV B deals with the transverse component.

\section{A. Longitudinal component}

The general expression for the adatom's enhanced longitudinal spin susceptibility [see Eq. (3)] within the TDDFT framework [47] is given by

$$
\chi_{\|}(\omega)=\frac{\chi_{\|}^{\mathrm{KS}}(\omega)}{1-U_{\|} \cdot \chi_{\|}^{\mathrm{KS}}(\omega)},
$$

where $U_{\|}$denotes the longitudinal XC kernel treated in the adiabatic local spin-density approximation [48] including the Coulomb term, while $\chi_{\|}^{\mathrm{KS}}(\omega)$ is the longitudinal KS spin susceptibility. We note that neglecting the direct contribution of the substrate atoms to the magnetic spin susceptibility is justified in the $\mathrm{Ag}$ and $\mathrm{Cu}$ substrates analyzed here since the polarizability of such elements is very weak [22-27].

In essence, $\chi_{\|}(\omega)$ in Eq. (5) describes the ability of the system to continuously modify the size of its magnetic moment by an externally applied time-dependent magnetic perturbation along the magnetization direction. The dynamics of this process can be phenomenologically studied in terms of the longitudinal Bloch equation, which yields the following form for the enhanced spin susceptibility [49] (see Appendix A),

$$
\chi^{\mathrm{Bl}}(\omega)=\frac{\chi_{0}^{\mathrm{Bl}}}{1-i \omega T_{\|}} .
$$

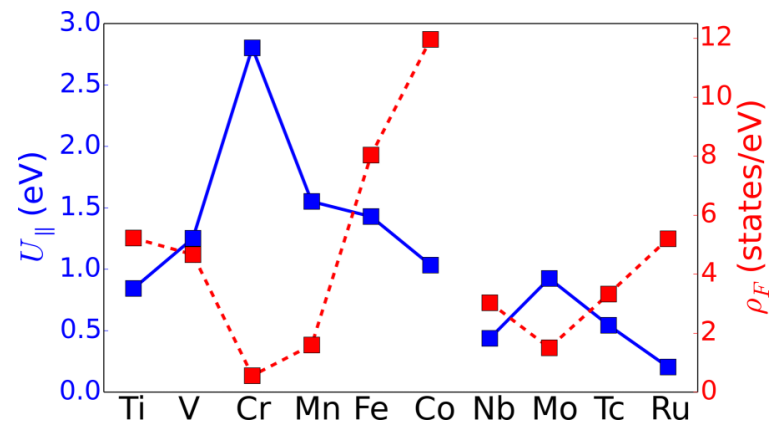

FIG. 3. $3 d$ and $4 d$ magnetic adatoms deposited on $\mathrm{Ag}(100)$. Solid (blue) and dashed (red) lines show the calculated values for $U_{\|}$[from Eq. (9)] and $\rho_{F}$ whose corresponding ordinate axes are placed on the left (blue) and right (red) of the graph, respectively. Note that lines are broken in order to separate $3 d$ from $4 d$ elements.

Above, $\chi_{0}^{\mathrm{Bl}}$ denotes a static spin susceptibility, while $T_{\|}$ corresponds to the longitudinal relaxation time mentioned in the introduction. Our aim is to establish a direct comparison between Eqs. (5) and (6). For this purpose, let us use the first-order Taylor expansion of the KS spin susceptibility [47]

$$
\chi_{\|}^{\mathrm{KS}}(\omega) \simeq \rho_{F}-i n_{e-h} \omega,
$$

with $\rho_{F}=\rho_{F, \uparrow}+\rho_{F, \downarrow}$ the DOS at the Fermi level and $n_{e-h}=$ $\pi\left(\rho_{F, \uparrow}^{2}+\rho_{F, \downarrow}^{2}\right) / 2$ the density of electron-hole excitations of the same spin channel. By inserting $\chi_{\|}^{\mathrm{KS}}(\omega)$ of Eq. (7) into Eq. (5), $\chi_{\|}(\omega)$ acquires a functional form in $\omega$ equal to that of $\chi^{\mathrm{Bl}}(\omega)$ in Eq. (6). This then allows us to obtain an expression for the longitudinal relaxation time in terms of basic electronic properties (see Appendix A for details):

$$
T_{\|}=\frac{U_{\|} n_{e-h}}{U_{\|} \rho_{F}-1} .
$$

The above expression is one of the main results of the present paper. First of all, it shows that the longitudinal relaxation time is mostly settled by the magnitude of electron-hole excitations weighted by the XC kernel [see the numerator of Eq. (8)]; this product is of order $1 / \mathrm{eV}$, hence settling the overall time scale of $T_{\|}$as fs. Secondly, it shows that $T_{\|}$diverges as $U_{\|} \rho_{F} \rightarrow 1$ (see the unitless denominator in the equation) and hence its magnitude can be strongly modified in this limit, i.e., as the system approaches the magnetic transition point. This feature reveals that weakly magnetic adatoms or even nonmagnetic adatoms close to the transition point can host long-living longitudinal excitations [47]. In the following, we first focus on quantitatively analyzing the ingredients of Eq. (8) and subsequently turn to $T_{\|}$itself.

In order to compute reliable values for the kernel $U_{\|}$, we make use of the static limit of Eq. (5), from which

$$
U_{\|}=\rho_{F}^{-1}-\chi_{\|}^{-1}(0) \text {. }
$$

We note that $\chi_{\|}(0)$ can be calculated by a standard ground state DFT calculation with a static magnetic field $\Delta B$ via $\chi_{\|}(0)=$ $\Delta m / \Delta B$, with $\Delta m$ the corresponding self-consistent change of the magnetic moment [50]. In Fig. 3 we show the calculated values of $\rho_{F}$ and $U_{\|}$for several $3 d$ and $4 d$ adatoms deposited on $\operatorname{Ag}(100)$. The most important message exposed by this figure is the large variation of $U_{\|}$among different elements; 


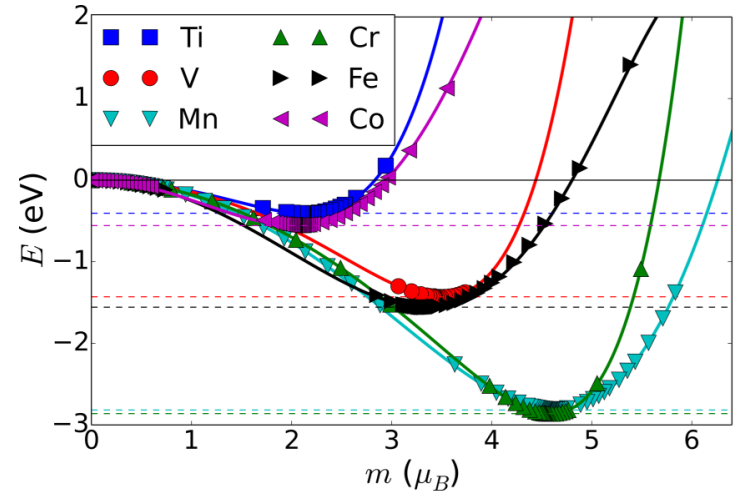

(a)

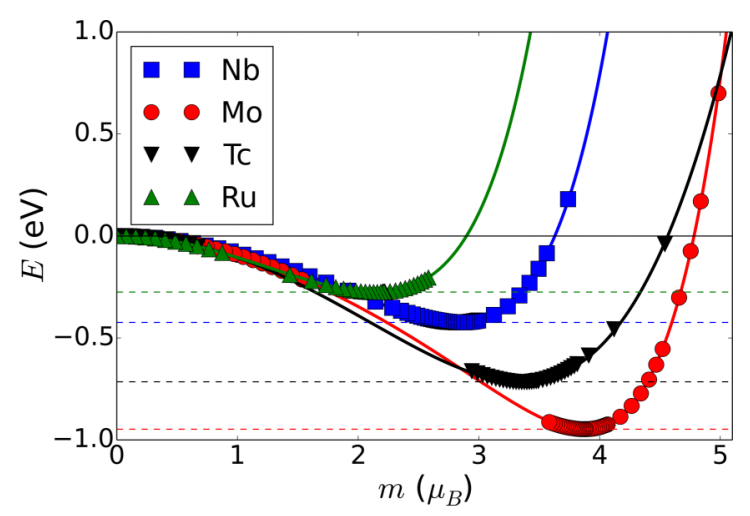

(b)

FIG. 4. (a) and (b), calculated energy as a function of the adatom's magnetic moment for $3 d$ and $4 d$ transition metal elements, respectively. DFT calculations are denoted by markers, while the solid lines are a fit to the calculations using $E=\sum_{i=2}^{8} a_{i} m^{i}$ with only even powers. The horizontal dashed lines mark the minimum energies for different adatoms.

while $U_{\|} \lesssim 0.5 \mathrm{eV}$ for most $4 d$ elements, $U_{\|} \gtrsim 1.5 \mathrm{eV}$ for various $3 d$ elements, reaching a maximum of one order of magnitude difference between $\mathrm{Ru}$ and $\mathrm{Cr}$. A second important feature revealed by Fig. 3 is the distribution of $U_{\|}$within each $d$ shell, whereby it is smallest at the ends of the row-case of $\mathrm{Ti}$ and $\mathrm{Co}$ among $3 d, \mathrm{Nb}$ and $\mathrm{Ru}$ among $4 d$ - and highest in the middle of the row-case of $\mathrm{Cr}$ and Mn among 3d, Mo and $\mathrm{Tc}$ among $4 d$-yielding an approximate inverted $\mathrm{V}$ shape. We note that $\rho_{F}$ in Fig. 3 shows the opposite behavior, i.e., it is minimum for $\mathrm{Cr}$ and maximum for $\mathrm{Co}$ and $\mathrm{Ru}$. This is consistent with Eq. (9), although we note strong deviations from the $U_{\|} \propto \rho_{F}^{-1}$ relationship (see in particular the case of $\mathrm{Cr}$ ), revealing the importance of the term $\chi_{\|}^{-1}(0)$ in Eq. (9).

The inverse of the static spin susceptibility $\chi_{\|}^{-1}(0)$ is closely connected to the magnetic equation of state, i.e., the dependence of the energy $E$ as a function of the magnetic moment $m$, via [50],

$$
\chi_{\|}^{-1}(0)=\left.\frac{\partial^{2} E(m)}{\partial m^{2}}\right|_{m=m_{0}}<0 .
$$

In essence, the magnetic equation of state informs about how stable the magnetic solution is in comparison to the nonmagnetic one. We have calculated $E(m)$ for the set of adatoms considered in Fig. 3 by employing DFT for fixed magnetic fields [50]. The results are shown in Fig. 4, which reveals that the energy difference between the magnetic and nonmagnetic state,

$$
\Delta E=E(m=0)-E\left(m=m_{0}\right),
$$

is of the order of $\mathrm{eV}$ and can largely vary for different adatoms. Importantly, our calculations show that $3 d$ adatoms overall have a substantially larger $\Delta E$ than $4 d$ adatoms; for $\mathrm{Cr}$, for instance, $\Delta E \sim 3 \mathrm{eV}$, while for Ru $\Delta E \sim 0.25 \mathrm{eV}$. Therefore, DFT predicts most $3 d$ adatoms to be magnetically more stable than $4 d$ ones, as expected. Furthermore, given that Eq. (10) together with Eq. (9) relates the XC kernel to the second derivative of the equation of state at $m_{0}$, one can establish an approximate connection between the depth of the minimum of $E(m)$ and the value of $U_{\|}$, as it is visible from the comparison of Figs. 4 and 3; the deeper the minimum, the larger $U_{\|}$.
We note that following the above procedure, one can also extract the kernel in the nonmagnetic ground state, i.e., the so-called Stoner XC parameter $I_{x c}$ [51]. This can be achieved by considering the curvature of $E(m)$ not at $m=m_{0}$ but at $m=0$, as well as using the nonmagnetic DOS in Eq. (9) instead of the magnetic one. As it is clearly visible from Fig. 4, the curvature is very different at $m=0$ and $m=m_{0}$. Furthermore, $\rho_{F}$ can also strongly vary from a magnetic to a nonmagnetic calculation. As a consequence, the distribution of $I_{x c}$ along the transition metal series first reported by Janak in Ref. [51] is very different from that of $U_{\|}$illustrated in Fig. 3 .

Having analyzed the properties of $\rho_{F}$ and $U_{\|}$, we next focus on the longitudinal relaxation time $T_{\|}$. The values calculated from Eq. (8) are plotted in Fig. 5 for $3 d$ and $4 d$ adatoms deposited on $\mathrm{Ag}(100)$ and $\mathrm{Cu}(111) . T_{\|}$is of the order of a few fs in all cases, being overall slightly larger for $4 d$ than $3 d$ adatoms, while the choice of substrate does not substantially affect it. Within each $d$ shell, $T_{\|}$is largest at the ends of the row while it is minimum for the half filled elements, thus resembling the behavior of $\rho_{F}$ (compare Figs. 3 and 5). Ru on $\operatorname{Ag}(100)$ has the highest value of $T_{\|} \sim 50 \mathrm{fs}$, mainly as a consequence of the denominator of Eq. (8) being closer to zero than in other elements. In contrast, $\mathrm{Cr}$ and $\mathrm{Mn}$ have $T_{\|} \sim$ 1 fs in both substrates, i.e., nearly two orders of magnitude

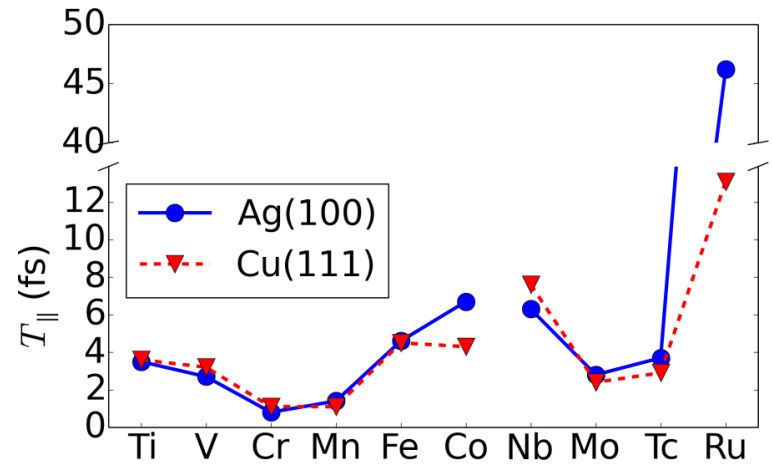

FIG. 5. Calculated values for the longitudinal relaxation time $T_{\|}$for magnetic $3 d$ and $4 d$ adatoms on $\operatorname{Ag}(100)$ (blue circles) and $\mathrm{Cu}(111)$ (red triangles). 
less than the aforementioned example. As a general feature, we note that the order of magnitude of $T_{\|}$is settled by the energy scale of the problem: All quantities involved in Eq. (8) are of the order of $\mathrm{eV}$, whose corresponding time scale is fs. Therefore, the longitudinal relaxation of the spin analyzed in this work is extremely fast. The physical reason is the large exchange splitting dominating the relaxation process, which makes it energetically very expensive to modify the length of the moment due to the high energies involved.

\section{Connection to experimental measurements}

Let us next consider the experimental scenario regarding the measurement of the longitudinal spin relaxation time. For this, we first note that in an experiment, several different mechanisms can contribute to this relaxation process, whose overall relaxation time is generally denoted as $T_{1}$. In this context, $T_{\|}$calculated here is a particular contribution to $T_{1}$, which may include further contributions depending on the physical processes taking place. To the best of our knowledge, the first experimental technique that measures $T_{1}$ in magnetic single adatoms was developed by Loth and co-workers [19]. Within this STM-based technique, the spin relaxation time was measured by monitoring the decay of electrons in excited states after the application of an all-electronic pump-probe scheme [19]. It is noteworthy that this scheme has so far only been applied to adatoms deposited on semi-insulating substrates, which are close to the atomic limit. The original work by Loth and co-workers measured $T_{1} \sim 90 \mathrm{~ns}$ for a Fe-Cu dimer on $\mathrm{Cu}_{2} \mathrm{Ni} / \mathrm{Cu}(100)$ [19]. A subsequent work by Rau and co-workers measured $T_{1} \sim 200 \mu$ s for a single Co atom on $\mathrm{MgO} / \mathrm{Ag}(100)$ [5]. Lastly, Baumann and co-workers reported $T_{1} \sim 90 \mu \mathrm{s}$ for a single $\mathrm{Fe}$ atom on $\mathrm{MgO} / \mathrm{Ag}(100)$ [14], while in a recent work of Paul and co-workers on the same system [52], the value of $T_{1}$ was enhanced up to the ms regime by fine tuning external conditions such as the height of the STM tip. To conclude, we note that the reported time resolution of the measuring technique employed in the above experiments ranges between a few ns to hundreds of ps.

All the above measured values of the spin relaxation time are several orders of magnitude larger than the values of order fs that we have calculated in this paper for $T_{\|}$(see Fig. 5). Let us first note that all of the above experiments are performed under externally applied static magnetic fields that range between $1 \mathrm{~T}$ and $10 \mathrm{~T}$. This, in turn, breaks the degeneracy of the spin ground states [19], a situation that is commonly modeled by a shifted discrete energy diagram as the one shown in Fig. 6(a). We note that excitations within such a diagram are not allowed to change the length of the spin moment (spin quantum number $S$ in this context) but only its projection (magnetic quantum number $S_{z}$ ). Therefore, the main spin relaxation process contributing to $T_{1}$ within such an scheme involves transitions between the two nondegenerate states with same $S$ but opposite $S_{z}$ [see Fig. 6(a)]. We note that their energy separation is of order meV, hence much smaller than the excitations of order $\mathrm{eV}$ involved in the change of the spin magnetic moment size considered for our calculation of $T_{\|}$, as schematically depicted in Fig. 6(b). On top of that, given that direct transitions between the two nondegenerate states of Fig. 6(a) are virtually inexistent, spin relaxation in
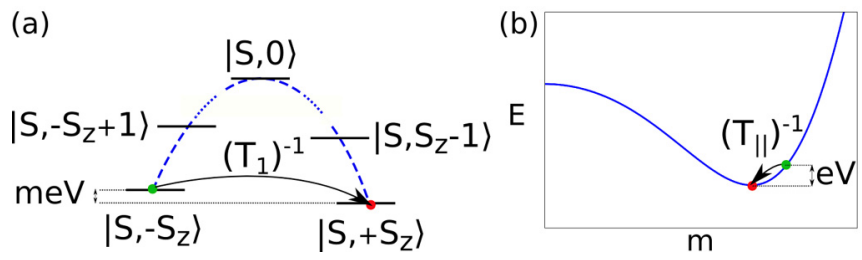

FIG. 6. Schematic illustration of two different processes contributing to the spin relaxation time. Green and red dots, respectively, represent the excited and ground states. (a) illustrates the prototypical energy diagram used to describe the experiments of Refs. [5,14,19,52]. The initially degenerate two ground states of maximum $S_{z}$ become nondegenerate by energy $\sim \mathrm{meV}$ under externally applied magnetic fields of 1-10 T. Transitions between these two states then determine $T_{1}$, which take place via quantum tunneling. (b) schematically describes the excitations contributing to $T_{\|}$considered in this paper. These take place in a continuum energy landscape of order $\mathrm{eV}$ and are driven by direct spin-conserving electron-hole transitions.

these conditions is driven by quantum tunneling processes, which are intrinsically much slower than the direct transitions considered in this paper. These two considerations explain why the spin relaxation time measured under the mentioned experimental conditions is several orders of magnitude larger than the values of $T_{\|}$obtained in this paper.

It is apparent that, in order to experimentally access the dynamics encoded into $T_{\|}$, a measuring scheme based on ultrafast techniques that modify the length of the spin magnetic moment is required. Considering the technological developments within STM measuring techniques [38-40], accessing the fs time scale of magnetic adatoms seems to be a reasonable goal for the near future by, e.g., using ultrafast laser pulses, a breakthrough that would allow us to monitor the ultrafast spin dynamics analyzed in this paper.

\section{B. Transverse component}

Unlike the longitudinal component, the transverse spin susceptibility and associated spin excitations of single adatoms have been thoroughly studied from first principles in, e.g., Refs. [22-27]. The general form for the adatom's enhanced transverse spin susceptibility [see Eq. (4)] in the TDDFT scheme is

$$
\chi_{ \pm}(\omega)=\frac{\chi_{ \pm}^{\mathrm{KS}}(\omega)}{1-U_{\perp} \chi_{ \pm}^{\mathrm{KS}}(\omega)} .
$$

Above, $U_{\perp}$ is the transverse $\mathrm{XC}$ kernel treated in the adiabatic local spin-density approximation [53], while $\chi_{ \pm}^{\mathrm{KS}}$ denotes the transverse KS spin susceptibility. Despite the formal similarity between Eq. (5) for $\chi_{\|}(\omega)$ and Eq. (12) for $\chi_{ \pm}(\omega)$, the underlying physics behind both expressions is very different. While the former contains excitations that modify the spin density, the latter describes damped precessional motion of the spin moment [49]. This motion, in turn, is described by the imaginary part of the enhanced spin susceptibility of Eq. (12), $\operatorname{Im} \chi_{ \pm}(\omega)$, which gives access to the density of transverse spin excitations of single adatoms [22-27].

The characteristic form of the spin excitation hosted by $\operatorname{Im} \chi_{ \pm}(\omega)$ is illustrated in Fig. 7 and is characterized by two 


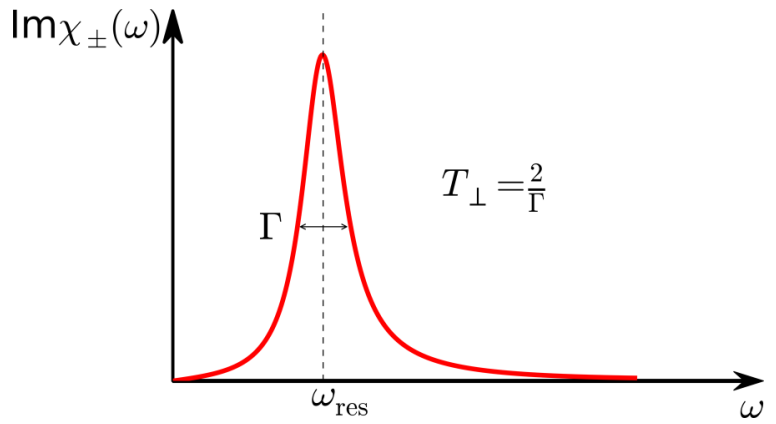

FIG. 7. Schematic illustration of a transverse spin excitation of a single adatom contained in $\operatorname{Im} \chi_{ \pm}(\omega)$ [see Eq. (12)]. The resonance frequency $\omega_{\text {res }}$ and the width $\Gamma$ are indicated in the figure.

main quantities. The first one is its resonance frequency, $\omega_{\text {res }}$, a fundamental property related to the magnetic anisotropy energy that is ultimately determined by SOC [24]. The second main quantity is the width of the spin excitation, $\Gamma$, which is proportional to the hybridization of the adatom's electrons with the substrate [22,23] (see also Ref. [54] for model Hamiltonian point of view). As shown in Ref. [25], the main contribution of the hybridization to $\Gamma$ is proportional to the electron-hole excitations of opposite spin channel, $n_{e-h}^{\prime}=\pi\left(\rho_{F, \uparrow} \cdot \rho_{F, \downarrow}\right)$ :

$$
\Gamma \simeq \frac{n_{e-h}^{\prime}}{\operatorname{Re} Q} \omega_{\text {res }}
$$

with $Q=\partial \chi_{ \pm}^{\mathrm{KS}}(\omega) /\left.\partial \omega\right|_{\omega=0}$. We note that the order of magnitude of $\omega_{\text {res }}$ ranges between $10^{-2}-1 \mathrm{meV}$ while $n_{e-h}^{\prime} / \operatorname{Re} Q$ is a unitless fraction that is typically of order unity.

Importantly, a finite width corresponds to a finite damping of the precessing magnetic moment and is thus directly linked to the transverse spin relaxation time [see Appendix B and Eq. (13)]:

$$
T_{\perp}=\frac{2}{\Gamma} \propto\left(n_{e-h}^{\prime}\right)^{-1} .
$$

We note that, while $T_{\|}$in Eq. (8) is directly proportional to the density of spin-conserving electron-hole excitations $n_{e-h}$, $T_{\perp}$ above is inversely proportional to the spin-flip counterpart $n_{e-h}^{\prime}$.

Using the TDDFT formalism developed in Refs. [22-24], we have calculated $T_{\perp}$ from the spin-excitation width for various $3 d$ and $4 d$ magnetic adatoms deposited on the metallic substrates $\mathrm{Ag}(100)$ and $\mathrm{Cu}(111)$; calculated values are shown in Fig. 8. One notes that the variation of $T_{\perp}$ among adatoms is considerably larger (up to two orders more) than that of $T_{\|}$, shown in Fig. 5. This is a consequence of the large variation of the width $\Gamma$ of atomic spin excitations, which can range from $10^{-2} \mathrm{meV}$ to a few meV [24,27], i.e., nearly three orders of magnitude change. This, in turn, can be linked to the electronic DOS at the Fermi level via Eq. (13); adatoms where the DOS peak of the $d$ states lies close to the Fermi level, case of Ti, $\mathrm{V}, \mathrm{Fe}$, and $\mathrm{Co}$ in Fig. 2, tend to be much more hybridized than those where only the tail of the DOS peak lies at the Fermi level, case of $\mathrm{Cr}$ and $\mathrm{Mn}$ in Fig. 2. In this way, $\mathrm{Cr}$ and Mn acquire large relaxation times of $T_{\perp} \sim 10^{1}-10^{2} \mathrm{ps}$, while strongly hybridized adatoms such as $\mathrm{Co}, \mathrm{Nb}$, and $\mathrm{Ru}$ have $T_{\perp} \sim 10^{-1}-10^{-2} \mathrm{ps}$.

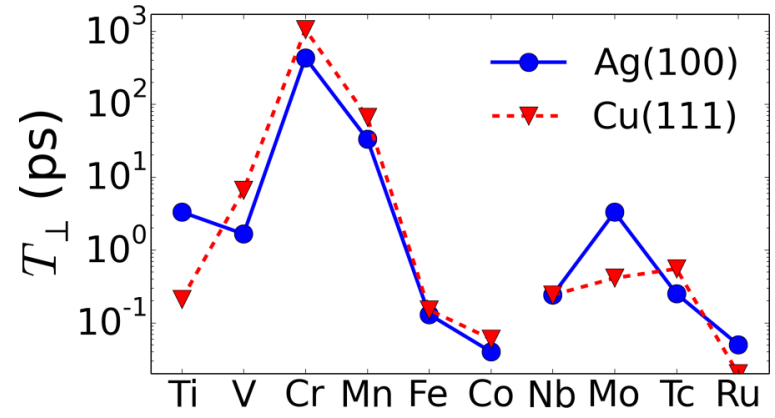

FIG. 8. Calculated values for the transverse relaxation time $T_{\perp}$ for magnetic $3 d$ and $4 d$ adatoms on $\mathrm{Ag}(100)$ (blue circles) and $\mathrm{Cu}(111)$ (red triangles). Note the logarithmic scale in the $y$ axis.

To conclude, let us note that the trend of $T_{\perp}$ within each $d$-shell row is opposite to that shown by $T_{\|}$(see Fig. 5). This comes as a consequence of the dependence on the density of electron-hole excitations, with $T_{\|} \propto n_{e-h}$ [see Eq. (8)] and $T_{\perp} \propto\left(n_{e-h}^{\prime}\right)^{-1}$ [see Eq. (13)]. In fact, $T_{\|}$and $T_{\perp}$ can be formally related to each other considering the relation between the spinconserving and spin-flip electron-hole contributions, namely $\rho_{F}^{2} / 2=n_{e-h}+n_{e-h}^{\prime}$. From Eq. (8) for $T_{\|}$and Eq. (13) for $T_{\perp}$ one can then infer the following expression:

$$
\frac{\rho_{F}^{2}}{2} \simeq T_{\|} \frac{U_{\|} \rho_{F}-1}{U_{\|}}+2 T_{\perp}^{-1} \frac{\operatorname{Re} Q}{\omega_{\text {res }}} .
$$

The unifying concept behind the above relationship between $T_{\|}$and $T_{\perp}$ is the hybridization of substrate electrons with the $d$ states of the transition metal adatoms, which in essence gives rise to a finite total $\rho_{F}$. However, despite the formal relationship, the fact that $T_{\|}$and $T_{\perp}$ in Eq. (15) have fundamentally different prefactors makes the time scale of the two relaxation constants differ by nearly three orders of magnitude.

\section{Connection to experimental measurements and a comment on nomenclature}

Next, we consider several experimental measurements of spin-excitation lifetimes of different single adatoms and connect them to our work. The lifetime of an atomic spin excitation can be experimentally accessed from the width of the step observed in IETS $d I / d V$ measurements, which provides a measure of $\Gamma$. Given that the energy resolution of this technique is $10^{-1} \mathrm{meV}$ at best [10], the longest lifetimes that can be inferred following this procedure are of order $10 \mathrm{ps}$ [see Eq. (14)]. These type of experiments can measure adatoms deposited on both metallic and semi-insulating substrates; as a general trend, the latter induce a larger lifetime than the former due to a far smaller electronic hybridization. We begin by considering Ref. [17], where Khajetoorians and co-workers estimate the spin-excitation lifetime of an Fe adatom deposited on metallic $\mathrm{Cu}(111)$ to be $0.2 \mathrm{ps}$, in very good quantitative agreement with our calculated value $T_{\perp}=0.15 \mathrm{ps}$ for the same system (see Fig. 8). Noteworthily, when the same atom is deposited on metallic $\mathrm{Pt}(111)$, the measured lifetime is increased by nearly an order of magnitude [10]. We note that we have found a similar variation between the two substrates considered in this work for the elements $\mathrm{Ti}, \mathrm{V}, \mathrm{Cr}$, and Mo, as it 
can be checked in Fig. 8. Focusing next on the semi-insulating $\mathrm{Cu}_{2} \mathrm{Ni} / \mathrm{Cu}(100)$ substrate, a lower bound of $\sim 10$ ps has been experimentally estimated for Fe [15,55], Mn [55], and Co [16] adatoms, although it is possible that the actual lifetimes are substantially larger. In fact, our calculations on $\mathrm{Cr}$ and $\mathrm{Mn}$, which are the elements with smallest hybridization and thus the ones closest to the semi-insulating limit, show that $T_{\perp}$ can reach up to $10^{3}$ ps (see Fig. 8); hence, it is not unlikely that the lifetimes of the aforementioned adatoms on $\mathrm{Cu}_{2} \mathrm{Ni} / \mathrm{Cu}(100)$ could be of the same order of magnitude. Last, it is worth noting the case of $\mathrm{Co}$ on $\mathrm{MgO}$ [5], which, despite being a semi-insulating substrate, yields a relatively short spin-excitation lifetime of $\sim 0.5 \mathrm{ps}$, i.e., a common value for adatoms deposited in metallic substrates analyzed in this paper (see Fig. 7).

To conclude this section, we note that the convention followed in the standard literature to denote the transversal relaxation time is $T_{2}$ (see, e.g., Refs. [49,56]). We have noticed, however, that the relaxation time associated to atomic spin excitations has in some cases been named as a $T_{1}$-like term; see, e.g., the review by Delgado and Fernández-Rossier [36]. The authors of this review use $T_{2}$ to denote another relaxation mechanism named as adiabatic decoherence. We have included a brief discussion on this nomenclature issue in Appendix C.

\section{CONCLUSIONS}

In conclusion, we have presented a systematic $a b$ initio investigation of longitudinal and transverse spin relaxation times of magnetic single adatoms deposited on metallic substrates. Our analysis has yielded as a main result the fact that the longitudinal spin relaxation process of single adatoms is much faster than the transverse one, i.e., $T_{\perp} \gg T_{\|}$. This, in turn, comes as a consequence of the energy scale of the corresponding processes; $\mathrm{eV}$ for $T_{\|}, \mathrm{meV}$ for $T_{\perp}$. Importantly, the two processes are triggered by different mechanisms: While $T_{\|}$is driven by spin-conserving excitations that change the spin density, $T_{\perp}$ depends on the atomic spin-flip spin excitations that induce the precessional motion. The comparison of our results with available experimental measurements shows that the relaxation times extracted from inelastic scanning tunneling spectroscopy $d I / d V$ curves show overall the same order of magnitude as $T_{\perp}$ and agree remarkably well in specific cases such as $\mathrm{Fe}$ on $\mathrm{Cu}(111)$ [17]. Regarding the measurement of $T_{\|}$, we have argued that, although currently available techniques cannot monitor the femtosecond regime of magnetic single adatoms, it is reasonable that this can be achieved in the near future, e.g., by employing STM-integrated ultrafast laser schemes [38-40], thus giving access to the ultrafast spin dynamics described in this paper.

\section{ACKNOWLEDGMENTS}

The authors are very grateful to F. Guimarães for fruitful discussions on the LLG model. This work has been supported by the Impuls und Vernetzungsfonds der HelmholtzGemeinschaft Postdoc Programme and funding from the European Research Council (ERC) under the European Union's Horizon 2020 research and innovation programme (ERC-consolidator Grant No. 681405 DYNASORE). The authors gratefully acknowledge the computing time granted by the JARA-HPC Vergabegremium and provided on the JARA-HPC Partition part of the supercomputer JURECA at Forschungszentrum Jülich.

\section{APPENDIX A: LONGITUDINAL BLOCH EQUATION}

The Bloch equation for the longitudinal change of the magnetization under the effect of a time-dependent perturbation $H_{1}(t)$ along the longitudinal direction can be written as [49]

$$
\frac{d m_{z}(t)}{d t}=\frac{\chi_{0}^{\mathrm{Bl}} H_{1}(t)-m_{z}(t)}{T_{\|}},
$$

with $\chi_{0}^{\mathrm{Bl}}$ a static spin susceptibility. The above equation describes how $m_{z}(t)$ comes back to equilibrium with a characteristic relaxation time $T_{\|}$after being perturbed by $H_{1}(t)$. Using $f(t)=\int d \omega f(\omega) e^{-i \omega t}$ for both $m_{z}(t)$ and $H_{1}(t)$ we can write Eq. (A1) in the frequency domain,

$$
\begin{aligned}
m_{z}(\omega)\left(-i \omega T_{\|}+1\right) & =\chi_{0} H_{1}(\omega) \Rightarrow \frac{m_{z}(\omega)}{H_{1}(\omega)} \\
& \equiv \chi^{\mathrm{Bl}}(\omega)=\frac{\chi_{0}^{\mathrm{Bl}}}{1-i \omega T_{\|}},
\end{aligned}
$$

where $\chi^{\mathrm{Bl}}(\omega)$ is the enhanced spin susceptibility. The real and imaginary parts of the above equation read

$$
\begin{aligned}
& \operatorname{Re} \chi^{\mathrm{Bl}}(\omega)=\frac{\chi_{0}^{\mathrm{Bl}}}{1+\left(\omega T_{\|}\right)^{2}}, \\
& \operatorname{Im} \chi^{\mathrm{Bl}}(\omega)=\frac{\chi_{0}^{\mathrm{Bl}} \omega T_{\|}}{1+\left(\omega T_{\|}\right)^{2}}
\end{aligned}
$$

Next, we consider the Taylor expansion of the KS spin susceptibility [see Eq. (7) in the main text],

$$
\chi_{\|}^{\mathrm{KS}}(\omega) \simeq \rho_{F}-i n_{e-h} \omega .
$$

The first-order expansion coefficient $n_{e-h}=\pi\left(\rho_{F, \uparrow}^{2}+\rho_{F, \downarrow}^{2}\right) / 2$ has been calculated in the Supplemental Material of Ref. [47]. Inserting $\chi_{\|}^{\mathrm{KS}}(\omega)$ of Eq. (A5) into the definition of the TDDFT enhanced spin susceptibility $\chi(\omega)$ [see Eq. (5) of the main text], the imaginary part $\operatorname{Im} \chi_{\|}(\omega)$ can be cast in the following way,

$$
\begin{aligned}
\operatorname{Im} \chi_{\|}(\omega) & =\frac{n_{e-h} \omega}{\left(1-U_{\|} \rho_{F}\right)^{2}} \cdot \frac{1}{1+\left(\frac{U_{\|} n_{e-h} \omega}{1-U_{\|} \rho_{F}}\right)^{2}} \\
& =\frac{\omega T_{\|}}{\left(1-U_{\|} \rho_{F}\right) U_{\|}} \cdot \frac{1}{1+\left(\omega T_{\|}\right)^{2}} \\
& =\frac{\chi(0)}{U_{\|} \rho_{F}} \cdot \frac{\omega T_{\|}}{1+\left(\omega T_{\|}\right)^{2}},
\end{aligned}
$$

where in the last step we used the expression for the static spin susceptibility $\chi_{\|}(0)=\rho_{F} /\left(1-U_{\|} \rho_{F}\right)$ and we defined

$$
T_{\|}=\frac{U_{\|} n_{e-h}}{1-U_{\|} \rho_{F}},
$$

which is the result quoted in the main text in Eq. (8). 


\section{APPENDIX B: TRANSVERSE RELAXATION WITHIN THE LANDAU-LIFSHITZ-GILBERT EQUATION}

We consider the Landau-Lifshitz-Gilbert (LLG) equation describing the damped precessional motion of a magnetic moment placed in a static external magnetic field that has been perturbed by a time-dependent transverse magnetic field:

$$
\frac{d \mathbf{m}}{d t}=-\gamma \mathbf{m} \times \mathbf{B}^{\mathrm{ext}}+\eta \frac{\mathbf{m}}{m_{0}} \times \frac{d \mathbf{m}}{d t},
$$

with $\mathbf{B}^{\text {ext }}=B_{0} \hat{\mathbf{z}}+\mathbf{b}(t)$ and $\mathbf{b}(t)=\theta(t) \Delta B(\hat{\mathbf{x}}+\hat{\mathbf{y}})$, i.e., for $t<0$ the static field points in the $z$ direction while for $t>0$ a small transverse component is switched on. Note that the precession rate in Eq. (B1) is set by $\gamma$, while the relaxation is controlled by $\eta$, namely the damping term. Let us assume $\mathbf{m}=$ $m_{0} \hat{\mathbf{z}}$ for $t<0$. Then, linearizing the LLG equation yields the following equation of motion for the transverse components of the magnetization:

$$
\begin{aligned}
& \frac{d m_{x}}{d t}=-\gamma B_{0} m_{y}+\gamma m_{0} \Delta B-\eta \frac{d m_{y}}{d t}, \\
& \frac{d m_{y}}{d t}=-\gamma m_{0} \Delta B+\gamma B_{0} m_{x}+\eta \frac{d m_{x}}{d t} .
\end{aligned}
$$

Since the expected solution is a damped precession that relaxes towards the direction of the static magnetic field, we use the following ansatz corresponding to a circular precession that decays in time with a transverse relaxation time $T_{\perp}$ :

$$
\begin{aligned}
& m_{x}(t)=m_{x}(\infty)-A e^{-t / T_{\perp}} \cos \left(\omega_{0} t\right), \\
& m_{y}(t)=m_{y}(\infty)-A e^{-t / T_{\perp}} \sin \left(\omega_{0} t\right) .
\end{aligned}
$$

Plugging the above ansatz back into the LLG equation (B1) we get

$$
\begin{aligned}
& \frac{1}{T_{\perp}} \cos \left(\omega_{0} t\right)+\omega_{0} \sin \left(\omega_{0} t\right) \\
& =\frac{\gamma\left(-B_{0} m_{y}(\infty)+m_{0} \Delta B\right)}{A} \\
& \quad \times \gamma B_{0} \sin \left(\omega_{0} t\right)-\eta\left(\frac{1}{T_{\perp}} \sin \left(\omega_{0} t\right)-\omega_{0} \cos \left(\omega_{0} t\right)\right),
\end{aligned}
$$

$$
\begin{aligned}
\frac{1}{T_{\perp}} & \sin \left(\omega_{0} t\right)-\omega_{0} \cos \left(\omega_{0} t\right) \\
= & \frac{\gamma\left(B_{0} m_{x}(\infty)-m_{0} \Delta B\right)}{A} \\
& \quad-\gamma B_{0} \cos \left(\omega_{0} t\right)+\eta\left(\frac{1}{T_{\perp}} \cos \left(\omega_{0} t\right)+\omega_{0} \sin \left(\omega_{0} t\right)\right) .
\end{aligned}
$$

The above equations can only be satisfied if the coefficients in front of the time-dependent sines and cosines match. We then have (both equations give the same pair of relations)

$$
\begin{gathered}
\frac{1}{T_{\perp}}=\eta \omega_{0}, \\
\omega_{0}=\frac{\gamma B_{0}}{1+\eta^{2}}, \\
m_{x}(\infty)=m_{y}(\infty)=\frac{m_{0}}{B_{0}} \Delta B=\chi_{\perp}^{\mathrm{LLG}} \Delta B,
\end{gathered}
$$

where $\chi_{\perp}^{\mathrm{LLG}}$ is the static spin susceptibility and continuity of $m_{x}(t)$ and $m_{y}(t)$ at $t=0$ fixes $A=m_{x}(\infty)$. Importantly, Eq. (B8) shows that the transverse relaxation time is given by the product between the damping term $\eta$ and the characteristic frequency $\omega_{0}$.

We next turn to calculate the transverse dynamic spin susceptibility within the LLG model. For this, we consider the following Fourier transforms,

$$
\mathbf{b}(t)=\int \frac{d \omega}{2 \pi} e^{-\mathrm{i} \omega t} \mathbf{b}(\omega), \quad \mathbf{m}(t)=\int \frac{d \omega}{2 \pi} e^{-\mathrm{i} \omega t} \mathbf{m}(\omega) .
$$

Inserting the above expressions into the linearized equations (B2) and (B3) we obtain in frequency space

$$
\begin{aligned}
& -i \omega m_{x}(\omega)=+\gamma m_{0} b_{y}(\omega)+\left(i \eta \omega-\gamma B_{0}\right) m_{y}(\omega), \\
& -i \omega m_{y}(\omega)=-\gamma m_{0} b_{x}(\omega)+\left(\gamma B_{0}-i \eta \omega\right) m_{x}(\omega) .
\end{aligned}
$$

The above can be simplified by considering the circular components $m_{ \pm}=m_{x} \pm i m_{y}$, yielding

$$
\begin{aligned}
-i \omega m_{ \pm}(\omega) & =\gamma_{ \pm}\left(B_{0} m_{ \pm}(\omega)-m_{0} b_{ \pm}(\omega)\right), \\
& \Rightarrow \Lambda_{ \pm}(\omega) m_{ \pm}(\omega)=b_{ \pm}(\omega),
\end{aligned}
$$

with $b_{ \pm}(\omega)=b_{x} \pm i b_{y}, \gamma_{ \pm}= \pm i \gamma /(1 \mp i \eta)$ and

$$
\Lambda_{ \pm}(\omega)=\frac{1}{\gamma_{ \pm} m_{0}}\left(i \omega+\gamma_{ \pm} B_{0}\right) \text {. }
$$

It is apparent from Eq. (B14) that the transverse spin susceptibility can be obtained from the inverse of $\Lambda_{ \pm}(\omega)$ defined above. After some algebra and picking the minus sign in Eq. (B15) one obtains

$$
\chi_{ \pm}^{\mathrm{LLG}}(\omega)=\left(\Lambda_{-}(\omega)\right)^{-1}=\frac{m_{0} \omega_{0}}{B_{0}} \frac{-\omega+\left(1+\eta^{2}\right) \omega_{0}+i \eta \omega}{\left(\omega-\omega_{0}\right)^{2}+\left(\eta \omega_{0}\right)^{2}} .
$$

The density of spin excitations in the LLG model are thus described by a skewed Lorentzian in $\omega$ :

$$
\operatorname{Im} \chi_{ \pm}^{\mathrm{LLG}}(\omega)=\frac{m_{0} \omega_{0}}{B_{0}} \frac{\eta \omega}{\left(\omega-\omega_{0}\right)^{2}+\left(\eta \omega_{0}\right)^{2}} .
$$

The resonance frequency of the above function takes place at

$$
\frac{d}{d \omega} \operatorname{Im} \chi_{ \pm}^{\mathrm{LLG}}(\omega)=0 \Rightarrow \omega_{\mathrm{res}}=\sqrt{1+\eta^{2}} \omega_{0},
$$

while the FWHM amounts to

$$
\Gamma=2 \eta \omega_{0} \frac{\sqrt{2+3 \eta^{2}+2 \sqrt{1+\eta^{2}}}}{1+\sqrt{1+\eta^{2}}} \simeq 2 \eta \omega_{0} .
$$

We note that the above approximation is exact in the $\eta \rightarrow 0$ limit and involves only a $\sim 10 \%$ relative error for $\eta=1$, which is by far the maximum value that damping can get for single adatoms; for most of the elements analyzed in the main text we have $\eta \lesssim 0.5$ [27], so the approximation of Eq. (B19) is indeed very good. Then, comparing Eq. (B19) to Eq. (B8) we arrive to the relation between the FWHM and the transverse relaxation time quoted in the main text:

$$
T_{\perp}=\frac{2}{\Gamma}
$$




\section{APPENDIX C: BASIC EXPRESSIONS OF THE BLOCH-REDFIELD FORMALISM}

In this Appendix we provide a brief summary of the relaxation times in the context of Bloch-Redfield (BR) theory (see Ref. [36] for details) in order to clarify the nomenclature regarding the relaxation time associated to a spin excitation. We begin with the longitudinal spin relaxation time, which in the BR theory describes the decay rate of diagonal matrix elements of the reduced density operator and is given by the following expression:

$$
\frac{1}{T_{1}} \equiv \Gamma_{n m}=2 \sum_{\alpha \beta} \operatorname{Re}\left(g_{\alpha \beta}\left(\omega_{m n}\right)\right) S_{\alpha}^{n m} S_{\beta}^{m n},
$$

where $n, m$ label the electronic eigenstates of the adatom, $\alpha, \beta$ label the eigenstates of the substrate, $\omega_{m n}=\epsilon_{m}-\epsilon_{n}$ with $\epsilon_{i}$ the eigenenergies, $g_{\alpha \beta}\left(\omega_{m n}\right)$ is the substrate operator correlator, and $S_{\alpha}^{n m}$ the matrix elements of the adatom's spin operator. Note that the term $\Gamma_{n m}$ in Eq. (C1) corresponds to the scattering rate from state $n$ to $m$, hence $T_{1}$ is associated to population transfer between different states. $T_{2}$, in turn, is termed as the decoherence time and describes the decay rate of off-diagonal matrix elements of the reduced density operator. It can be separated into two different contributions, namely the nonadiabatic one, $\gamma_{n m}^{\text {nonad }}$, and the adiabatic one, $\gamma_{n m}^{\mathrm{ad}}$,

$$
\frac{1}{T_{2}}=\gamma_{n m}^{\mathrm{nonad}}+\gamma_{n m}^{\mathrm{ad}}
$$

The nonadiabatic contribution is given by

$$
\gamma_{n m}^{\text {nonad }}=\frac{1}{2}\left(\sum_{n^{\prime} \neq n} \Gamma_{n n^{\prime}}+\sum_{n^{\prime} \neq m} \Gamma_{m n^{\prime}}\right),
$$

while the adiabatic one reads

$$
\gamma_{n m}^{\mathrm{ad}}=\frac{1}{2} \sum_{\alpha \beta} \operatorname{Re}\left(g_{\alpha \beta}(0)\right)\left(S_{\alpha}^{m m}-S_{\alpha}^{n n}\right)\left(S_{\beta}^{m m}-S_{\beta}^{n n}\right) .
$$

Noteworthily, the relaxation time of an atomic spin excitation in the BR theory is described by $\gamma_{n m}^{\text {nonad }}$ of Eq. (C3). The most important aspect to note for our purpose here is that $\gamma_{n m}^{\text {nonad }}$ in Eq. (C3) involves a population transfer $\Gamma_{n n^{\prime}}$, while $\gamma_{n m}^{\text {ad }}$ in Eq. (C4) does not. As a consequence, $\gamma_{n m}^{\text {nonad }}$ and hence the spin-excitation lifetime is regarded as a $T_{1}$-like term (see Eqs. (69) and (70) of Ref. [36]), even though it formally describes the decay rate of off-diagonal matrix elements of the density operator rather than diagonal ones. Meanwhile, $\gamma_{n m}^{\text {ad }}$ is named the pure decoherence contribution [36]. This, in our understanding, is how and why the relaxation time of an atomic spin excitation is associated to $T_{1}$ instead of $T_{2}$ in this context.

We note that the above convention is not in line with the one adopted in the present paper. From our point of view, given that an atomic spin excitation can be related to the damped precessional (transversal) motion of the adatom's magnetic moment, it is more natural to denote its lifetime by $T_{2}$ instead of with $T_{1}$.
[1] A. Oswald, R. Zeller, and P. H. Dederichs, Phys. Rev. Lett. 56, 1419 (1986).

[2] K. Wildberger, V. S. Stepanyuk, P. Lang, R. Zeller, and P. H. Dederichs, Phys. Rev. Lett. 75, 509 (1995).

[3] P. Lang, V. S. Stepanyuk, K. Wildberger, R. Zeller, and P. H. Dederichs, Solid State Commun. 92, 755 (1994).

[4] P. Gambardella, S. Rusponi, M. Veronese, S. S. Dhesi, C. Grazioli, A. Dallmeyer, I. Cabria, R. Zeller, P. H. Dederichs, K. Kern et al., Science 300, 1130 (2003).

[5] I. G. Rau, S. Baumann, S. Rusponi, F. Donati, S. Stepanow, L. Gragnaniello, J. Dreiser, C. Piamonteze, F. Nolting, S. Gangopadhyay et al., Science 344, 988 (2014).

[6] J. Honolka, A. A. Khajetoorians, V. Sessi, T. O. Wehling, S. Stepanow, J.-L. Mi, B. B. Iversen, T. Schlenk, J. Wiebe, N. B. Brookes et al., Phys. Rev. Lett. 108, 256811 (2012).

[7] A. J. Heinrich, J. A. Gupta, C. P. Lutz, and D. M. Eigler, Science 306, 466 (2004).

[8] C. F. Hirjibehedin, C. P. Lutz, and A. J. Heinrich, Science 312, 1021 (2006).

[9] B. W. Heinrich, L. Braun, J. I. Pascual, and K. J. Franke, Nano Lett. 15, 4024 (2015).

[10] A. A. Khajetoorians, T. Schlenk, B. Schweflinghaus, M. dos Santos Dias, M. Steinbrecher, M. Bouhassoune, S. Lounis, J. Wiebe, and R. Wiesendanger, Phys. Rev. Lett. 111, 157204 (2013).
[11] Q. Dubout, F. Donati, C. Wäckerlin, F. Calleja, M. Etzkorn, A. Lehnert, L. Claude, P. Gambardella, and H. Brune, Phys. Rev. Lett. 114, 106807 (2015).

[12] J. C. Oberg, M. R. Calvo, F. Delgado, M. Moro-Lagares, D. Serrate, D. Jacob, J. Fernández-Rossier, and C. F. Hirjibehedin, Nat. Nanotechnol. 9, 64 (2014).

[13] F. Donati, S. Rusponi, S. Stepanow, C. Wäckerlin, A. Singha, L. Persichetti, R. Baltic, K. Diller, F. Patthey, E. Fernandes et al., Science 352, 318 (2016).

[14] S. Baumann, W. Paul, T. Choi, C. P. Lutz, A. Ardavan, and A. J. Heinrich, Science 350, 417 (2015).

[15] C. F. Hirjibehedin, C.-Y. Lin, A. F. Otte, M. Ternes, C. P. Lutz, B. A. Jones, and A. J. Heinrich, Science 317, 1199 (2007).

[16] A. F. Otte, M. Ternes, K. von Bergmann, S. Loth, H. Brune, C. P. Lutz, C. F. Hirjibehedin, and A. J. Heinrich, Nat. Phys. 4, 847 (2008).

[17] A. A. Khajetoorians, S. Lounis, B. Chilian, A. T. Costa, L. Zhou, D. L. Mills, J. Wiebe, and R. Wiesendanger, Phys. Rev. Lett. 106, 037205 (2011).

[18] A. Stróżecka, A. Eiguren, and J. I. Pascual, Phys. Rev. Lett. 107, 186805 (2011).

[19] S. Loth, M. Etzkorn, C. P. Lutz, D. M. Eigler, and A. J. Heinrich, Science 329, 1628 (2010).

[20] F. D. Natterer, K. Yang, W. Paul, P. Willke, T. Choi, T. Greber, A. J. Heinrich, and C. P. Lutz, Nature (London) 543, 226 (2017).

[21] E. Runge and E. K. U. Gross, Phys. Rev. Lett. 52, 997 (1984). 
[22] S. Lounis, A. T. Costa, R. B. Muniz, and D. L. Mills, Phys. Rev. Lett. 105, 187205 (2010).

[23] S. Lounis, A. T. Costa, R. B. Muniz, and D. L. Mills, Phys. Rev. B 83, 035109 (2011).

[24] M. dos Santos Dias, B. Schweflinghaus, S. Blügel, and S. Lounis, Phys. Rev. B 91, 075405 (2015).

[25] S. Lounis, M. dos Santos Dias, and B. Schweflinghaus, Phys. Rev. B 91, 104420 (2015).

[26] B. Schweflinghaus, M. dos Santos Dias, A. T. Costa, and S. Lounis, Phys. Rev. B 89, 235439 (2014).

[27] J. Ibañez-Azpiroz, M. dos Santos Dias, S. Blügel, and S. Lounis, Nano Lett. 16, 4305 (2016).

[28] C. Hübner, B. Baxevanis, A. A. Khajetoorians, and D. Pfannkuche, Phys. Rev. B 90, 155134 (2014).

[29] A. A. Khajetoorians, B. Baxevanis, C. Hbner, T. Schlenk, S. Krause, T. O. Wehling, S. Lounis, A. Lichtenstein, D. Pfannkuche, J. Wiebe et al., Science 339, 55 (2013).

[30] N. Lorente and J.-P. Gauyacq, Phys. Rev. Lett. 103, 176601 (2009).

[31] J. Fernández-Rossier, Phys. Rev. Lett. 102, 256802 (2009).

[32] M. Persson, Phys. Rev. Lett. 103, 050801 (2009).

[33] J. Fransson, Nano Lett. 9, 2414 (2009).

[34] B. Sothmann and J. Knig, New J. Phys. 12, 083028 (2010).

[35] F. Delgado and J. Fernández-Rossier, Phys. Rev. B 82, 134414 (2010).

[36] F. Delgado and J. Fernández-Rossier, Prog. Surf. Sci. 92, 40 (2017).

[37] C. Saunus, J. Raphael Bindel, M. Pratzer, and M. Morgenstern, Appl. Phys. Lett. 102, 051601 (2013).

[38] M. Krüger, M. Schenk, and P. Hommelhoff, Nature (London) 475, 78 (2011).

[39] T. L. Cocker, V. Jelic, M. Gupta, S. J. Molesky, J. A. J. Burgess, G. D. L. Reyes, L. V. Titova, Y. Y. Tsui, M. R. Freeman, and F. A. Hegmann, Nat. Photonics 7, 620 (2013).
[40] T. L. Cocker, D. Peller, P. Yu, J. Repp, and R. Huber, Nature (London) 539, 263 (2016).

[41] N. Papanikolaou, R. Zeller, and P. H. Dederichs, J. Phys.: Condens. Matter 14, 2799 (2002).

[42] S. H. Vosko, L. Wilk, and M. Nusair, Can. J. Phys. 58, 1200 (1980).

[43] P. Giannozzi, S. Baroni, N. Bonini, M. Calandra, R. Car, C. Cavazzoni, Davide Ceresoli, G. L. Chiarotti, M. Cococcioni, I. Dabo et al., J. Phys.: Condens. Matter 21, 395502 (2009).

[44] H. T. Dang, M. dos Santos Dias, A. Liebsch, and S. Lounis, Phys. Rev. B 93, 115123 (2016).

[45] A. I. Liechtenstein, V. I. Anisimov, and J. Zaanen, Phys. Rev. B 52, R5467 (1995).

[46] G. F. Giuliani and G. Vignale, Quantum Theory of the Electron Liquid (Cambridge University Press, Cambridge, UK, 2005).

[47] J. Ibañez-Azpiroz, M. dos Santos Dias, B. Schweflinghaus, S. Blügel, and S. Lounis, Phys. Rev. Lett. 119, 017203 (2017).

[48] L. Ortenzi, I. I. Mazin, P. Blaha, and L. Boeri, Phys. Rev. B 86, 064437 (2012).

[49] R. M. White, Quantum Theory of Magnetism: Magnetic Properties of Materials (Springer-Verlag, Berlin, Heidelberg, 2007).

[50] J. Kübler, Theory of Itinerant Electron Magnetism, International Series of Monographs on Physics (Oxford University Press, Oxford, New York, 2009).

[51] J. F. Janak, Phys. Rev. B 16, 255 (1977).

[52] W. Paul, K. Yang, S. Baumann, N. Romming, T. Choi, C. P. Lutz, and A. J. Heinrich, Nat. Phys. 13, 403 (2017).

[53] M. I. Katsnelson and A. I. Lichtenstein, J. Phys.: Condens. Matter 16, 7439 (2004).

[54] M. Ternes, New J. Phys. 17, 063016 (2015).

[55] S. Loth, C. P. Lutz, and A. J. Heinrich, New J. Phys. 12, 125021 (2010).

[56] D. Canet and P. Mutzenhardt, in Relaxation in Nuclear Magnetic Resonance, General, Encyclopedia of Analytical Chemistry (John Wiley \& Sons, Ltd., Chichester, 2006). 